\title{
Análisis cultural y lingüístico de algunas particularidades de los sistemas alimentarios de Francia y Colombia
}

\author{
A cultural and linguistic analysis of some distinctive \\ characteristics of the food systems of France and Colombia
}

\author{
Olga Rocío Serrano Carranza \\ Departamento de Lenguas Modernas \\ Universidad $\mathrm{ECCl}$, Colombia
}

\section{Resumen}

Este artículo expone un estudio comparativo sobre la temática de la alimentación. Se enmarca dentro del proyecto Dico Culturel, que propone un modelo de diccionario francés-español de Colombia. En primer lugar, reflexionamos sobre conceptos clave de los sistemas alimentarios. Luego, por medio de la consulta de las fuentes bibliográficas, seleccionamos una muestra de palabras francesas y examinamos las informaciones lingüísticas y culturales asociadas, estableciendo paralelos con el español de Colombia y su cultura. Concluimos resaltando cómo, mediante el análisis textual, es posible encontrar informaciones de interés para la concepción del prototipo de diccionario cultural.

Palabras clave: cocinas, cultura, diccionario cultural, contenidos lingüísticos, diccionario bilingüe

\begin{abstract}
This article presents a comparative study of the subject of food. It is framed within the Dico Culturel project that seeks to create a prototype of a French-Colombian Spanish dictionary. In the first place, we discuss key concepts of the systems of food. Then, through a consultation of the bibliographical sources, we choose a sample of words in French and examine the items of linguistic and cultural information they contain, in order to establish parallels with Colombian Spanish and the culture of Colombia. We conclude by highlighting how, through textual analysis, it is possible to find multiple items of information of interest for designing the prototype of a cultural dictionary.
\end{abstract}


Keywords: cooking, culture, cultural dictionary, linguistic contents, bilingual dictionary

\section{Introducción}

$\mathrm{P}$ ara el proyecto de investigación Dico Culturel (Diccionario Cultural), subvencionado por la Universidad ECCI (Escuela Colombiana de Carreras Industriales), en Bogotá, Colombia, y desarrollado por la carrera de Lenguas Modernas y el grupo de investigación IP (Innovación Pedagógica), indagamos sobre temáticas generales o en áreas de especialidad con el fin de identificar y describir el léxico que transmite contenidos culturales variables entre Francia y Colombia, y que es útil conocer cuando se aprende el francés como lengua extranjera.

Los contenidos culturales hacen alusión a actitudes, comportamientos, creencias, rituales, tradiciones, formas de concebir la realidad, connotaciones sociales, representaciones simbólicas, maneras de funcionar, entre otros. Estos elementos culturales se inscriben en la sociedad francesa y colombiana contemporánea o se explican mediante cambios que han ocurrido a lo largo del tiempo.

En este artículo, nos centramos en la temática de la alimentación. A partir de la muestra de palabras francesas $b a$ guette, crêpe, galette, soupe y déjeuner, introducimos ejemplos, consideraciones y paralelos lingüísticos y culturales entre Francia y Colombia.

Se trata entonces de un breve examen de informaciones que contribuyen a elaborar el prototipo de diccionario cultural que propone un modelo bilingüe, unidireccional francés-español de
Colombia en el que se puntualizan lingüística, cultural e interculturalmente 100 entradas.

\section{Sistemas culturales alimentarios: ejemplos de Francia y Colombia}

Cada comunidad está sujeta a un conjunto de reglas universales y particulares relativas a la alimentación. Estas reglas están subordinadas a factores biológicos y a los recursos consumibles disponibles en los ambientes naturales en donde viven los individuos. El ser humano construye, pues, su propio sistema alimentario a partir de su hábitat, de los conocimientos que adquiere sobre este y de su capacidad de ser omnívoro (Fumey, 2008, p. 1).

Además de los aspectos biológicos y fisiológicos, los hábitos y sistemas de alimentación dependen de factores geográficos, históricos, sociales y religiosos que los convierten en elementos culturales inscritos en modelos de representaciones, símbolos y rituales en torno a los alimentos.

A diferencia de los animales que consumen directamente sustentos naturales disponibles en el medio ambiente, los seres humanos han desarrollado estrategias y técnicas para cultivarlos mediante la agricultura o criarlos, en el caso de los productos de origen animal. Estos se seleccionan, se incorporan a su dieta, se modifican y se cocinan para crear nuevos platos 0 comidas, regidos por pautas que dan 
lugar a hábitos o tradiciones (Goyan et al., 2015, p. 1). Todos estos procesos conforman lo que varios sociólogos, antropólogos y etnólogos llaman modelos culturales alimentarios.

Poulain (2002, p. 25) los define como sistemas estructurados que giran alrededor de códigos simbólicos, elementos y conocimientos sociales y técnicos, por medio de los cuales se crean y se transmiten valores dentro de los grupos sociales a lo largo del tiempo. Esto genera una identidad cultural alimentaria singular que distingue a las comunidades entre sí.

Para referirse a los modelos culturales alimentarios, otros autores los llaman "cocinas". Así, aparte de los significados generales atribuidos a este término, a saber, el de "pieza o sitio de la casa en el cual se guisa la comida" (Real Academia Española, 2016, en línea); el de "arte o manera especial de guisar de cada país y de cada cocinero" (Real Academia Española, 2016, en línea) o el de "conjunto de ingredientes y de técnicas utilizadas en la preparación de la comida" (Fischler, 1990, p. 31), la cocina puede ser explicada como una combinación de:

representaciones, creencias y prácticas que están asociadas a ella y que comparten los individuos que forman parte de una cultura o de un grupo en el interior de esta cultura. Cada cultura posee una cocina específica que implica clasificaciones, taxonomías particulares y un conjunto complejo de reglas que atienden no sólo a la preparación y combinación de alimentos, sino también a su cosecha y a su consumo. Posee igualmente significaciones que están en dependencia estrecha de la manera como se aplican las reglas culinarias (Fischler, 1995, p. 34).

Para Claude Lévi-Strauss, las cocinas son conocimientos adquiridos a través de múltiples experiencias de ensayo y error (Poulain, 2002, p. 27) que permiten al ser humano alimentarse adecuadamente (Fumey, 2008, p. 1). De modo semejante, las define como un lenguaje o código que pone de manifiesto "modelos sobre las relaciones sociales" (Lévi-Strauss, 1983, citado por Stajcic, 2013, p. 8) o como "una actividad intermedia entre la naturaleza y la cultura" (Lévi-Strauss, 1968, p. 405, citado por Verdier, 1969, p. 51) que "mediatiza las relaciones entre el hombre y el universo" (Lévi-Strauss, 1968, p. 421, citado por Verdier, 1969, p. 51).

Además de la noción de modelos culturales alimentarios o cocinas, otros autores hablan de ideología alimentaria y la entienden como una asociación de connotaciones, actitudes, creencias, símbolos y prohibiciones (Rasse y Debos, 2006, p. 2) alrededor de la cual quienes comen y lo que comen se ven influenciados (Eckstein, 1980, citado por Fieldhouse, 1995, p. 30).

La comida consumida en el interior de una cultura está sometida a normas y su selección e ingesta se ve motivada por una serie de imaginarios conscientes o inconscientes. Determinados alimentos poseen valoraciones positivas o negativas que provocan su rechazo en ciertas comunidades (Poulain, 2002, p. 27). Por tal razón, es probable sentir repugnancia hacia platos pertenecientes a una cultura diferente a la nuestra, ya que "tout ce qui est biologiquement mangeable n'est pas culturellement comestible" (Fischler, 1990, p. 31) 
. Esto puede ser ejemplificado con platos franceses como les escargots (los caracoles) o les cuisses de grenouille (las ancas de rana):

La primera vez que vi caracoles comestibles pensé que se trataba de una broma. Era una mañana de invierno [...], hace más de treinta años [...]. En una esquina había un hombre ofreciendo a los transeúntes papas fritas y caracoles en bolsitas de papel. Fue necesario observar con mis propios ojos a un burgués bigotudo extraer esos gusanos babosos con un palillo de sus conchas y llevárselos a la boca, para comprender la naturaleza humana (Allende, 2014, en línea).

Tal vez usted no ha tenido la oportunidad de comer ancas de rana o le repugna el solo hecho de pensarlo. Aquí le damos algunas razones para que se decida a probar este exótico platillo (Franco, 2000, en línea).

Otro aspecto que vale la pena mencionar es el hecho de comer alimentos crudos, casi crudos, cocidos o muy bien cocidos. Esto es esquematizado con el famoso triángulo culinario imaginado por Claude Lévi-Strauss en el que cada uno de los vértices representa las categorías "crudo" (vértice superior), "cocinado" (vértice inferior izquierdo) y "podrido" (vértice inferior derecho) (Lévi-Strauss, 2003, p. 428; Lévi-Strauss, 2009, p. 17). Los platos ingeridos por las poblaciones y las técnicas empleadas en su preparación se ubican y comparan con este modelo triangular. No obstante, cabe precisar que estas categorías no son concebidas idénticamente en las diversas culturas.
Un producto comestible que sirve de ilustración es la carne. En Francia, esta puede consumirse cruda. Esto ocurre con una comida llamada tartare en la que la carne se come molida y sin cocinar:

Beef or horse chopped up and eaten raw is "tartare". The delicate, sweet-tasting raw meat is garnished with pungent, highly flavored condiments, including chopped onion, mustard, salt, pepper, capers, [...], and parsley; sometimes a raw egg is broken over the tartare (Abramson, 2007, p. 46).

Las expresiones francesas viande bleue (carne azul) y viande saignante (carne sangrante) aluden también a la categoría de cocción "crudo": "Thicker steaks are eaten 'bleu' [...], 'saignant', or 'à point' [...], according to preference. Tastes do not run to well-cooked steaks. Overcooking dries out the meat, ruining the flavor as well as the texture (Abramson, 2007, p. 46).

En Colombia, se prefiere la carne cocida o bien cocida: "Aunque como entre gustos no hay disgustos, en otros países [...] la consumen a término azul o cruda. En Colombia, el 75 \% de las personas la elige a tres cuartos o bien asada, y el porcentaje restante a término medio (Santos, 2013, en línea).

Con respecto a la categoría de lo "podrido", hacemos referencia a diversos quesos de origen francés como el fromage bleu (queso azul) o el fromage Roquefort (queso Roquefort), a los que se les introducen cultivos de hongos (penicillium) durante su elaboración, los cuales provocan su color azuloso o verdoso. Para varios habitantes de Colombia, ver moho en estos alimentos provoca repugnancia o miedo a 
enfermarse. Por el contrario, muchos franceses consideran que, además de actuar como antibióticos naturales, estos hongos cumplen una función sensorial y gustativa: "Filamentous fungi also play a central role during cheesemaking. Two well-known fungal ripening cultures, Penicillium camemberti and Penicillium roqueforti, which play a significant role in appearance, texture, and flavor development of mold-ripened and blue-veined cheese" (Hymery et al., 2014, p. 438).

Las sociedades eligen entonces lo que incluyen o no en su dieta y el método de preparación de las comidas de origen vegetal o animal. Las categorizaciones positivas o negativas que se les atribuyen a los alimentos pueden ser implícitas, conscientes o inconscientes. También son de carácter psicológico, emocional, sociocultural, religioso, nutricional, económico o estético y se trasmiten de generación en generación (Fieldhouse, 1995, p. 33).

Por medio de estas reglas, los sistemas culturales alimentarios se afianzan y los individuos se sienten incluidos dentro de grupos socioculturales que se diferencian de otros, no solo a nivel nacional, sino también con respecto a otros países y culturas (Poulain, 2002, p. 27). Lo que comemos se convierte, por consiguiente, en un símbolo significativo de lo que somos e inversamente de lo que no somos (Goyan et al., 2015, p. 4) y nos identificamos con la comunidad de la que formamos parte porque comemos lo mismo de manera similar (Fox, 2003, p. 2). En efecto:

C'est sur les pratiques alimentaires, vitalement essentielles et quotidiennes, que se construit le sentiment d'appartenance ou de différence sociale. C'est par la cuisine et les manières de table que s'opèrent les apprentissages sociaux, les plus fondamentaux, et qu'une société transmet et permet l'intériorisation de ses valeurs. C'est par l'alimentation que se tissent et s'entretiennent les liens sociaux. Si l'aliment nourrit l'être biologique, l'aliment cuisiné, c'est-à-dire enculturé, nourrit, quant à lui, le « corps social» (Aron, 1997, pp.127-128).

Además de alimentar el organismo, las comidas cumplen funciones sociales de convivialidad, interacción y cohesión comunitaria (Goyan et al., 2015, p. 3) que se manifiestan mediante tradiciones, rituales o fiestas.

Finalmente, se observa que los modelos culturales alimentarios influyen en el número y horarios de las comidas (Poulain, 2002, p. 27), en el orden y la manera en la que estas y sus diferentes componentes son consumidos día a día. Igualmente, determinan la ocasión, el lugar y con quiénes estos alimentos deben ser preparados, servidos e ingeridos (Poulain, 2002, p. 24; Fox, 2003, p. 3; Goyan et al., 2015, p. 1).

Si vinculamos las ideas anteriores al proceso de enseñanza-aprendizaje de una lengua extranjera, constatamos que es indispensable meditar sobre la cultura que se asocia a esta, puesto que así comprenderemos mejor al "otro" y enriquecemos nuestras competencias comunicativas e interculturales.

\section{Selección de la muestra y colecta de información}

Con el fin de identificar el léxico sobre la temática de la alimentación, 
revisamos varios manuales y libros de civilización y cultura destinados al aprendizaje de la lengua francesa. La lista se muestra en el Anexo 1.

El vocabulario resaltado se refiere a alimentos brutos o elaborados, nombres de comidas, platos típicos, objetos que sirven para consumir los alimentos, marcas atribuidas a estos, comportamientos o reglas ligadas al comer, profesiones o establecimientos comerciales del sector alimentario, entre otros. Una muestra se detalla en el Anexo 2.

Aunque muchas de estas unidades lingüísticas y expresiones son potencialmente relevantes para ser examinadas comparativamente, las palabras elegidas para este artículo fueron $b a$ guette, crêpe, galette, soupe y déjeuner.

Uno de los criterios por el cual las seleccionamos es porque aparecían continuamente en los libros escrutados. Esto significa que son muy características de la lengua general y del vocabulario comúnmente enseñado a los estudiantes de lengua extranjera. Adicionalmente, algunas (baguette, crêpe y galette) son extremadamente representativas de Francia. Nos pareció interesante indagar sobre las razones de tal preponderancia.

Las demás (soupe y déjeuner), al formar parte de los dos universos culturales de manera más homogénea, posibilitan poner de manifiesto múltiples similitudes o divergencias, leves o pronunciadas, sobre las que no solemos reflexionar en clase de lengua porque nos limitamos a buscar una traducción sin ir más allá de lo que aportaría un análisis lingüístico o intercultural más exhaustivo.

Para recopilar las informaciones sobre la muestra de palabras francesas y los equivalentes en español de Colombia, digitamos en el formulario de exploración avanzada de Google (2018, en línea) la palabra clave junto con otras (p. ej. traditions, tradiciones; croyances, creencias; origine, origen; histoire, historia; consommation, consumo; France, Colombia; Français, colombianos, etc.). Para obtener únicamente datos de páginas de Francia y Colombia, delimitamos el país e idioma.

Las informaciones forman parte del rango temporal 1980-2018 y provienen esencialmente de artículos de periódicos, revistas, sitios de internet especializados, consagrados a la enseñanza del francés, páginas web institucionales, blogs o páginas personales. También proceden de obras en formato impreso o digital en inglés, francés o español, disponibles en Google Books (2018, en línea). Igualmente, examinamos ciertos diccionarios monolingües y bilingües (estos figuran en el Anexo 1).

Para ilustrar el análisis, nos fundamentamos primordialmente en fuentes fiables y cuando no era posible, en fuentes catalogadas como "no fiables" (p. ej. blogs o páginas personales). Pensamos que estas últimas, al ser escritas por hablantes nativos que están inmersos en su cultura respectiva, pueden dar cuenta de fenómenos lingüísticos y culturales auténticos y útiles.

Presentamos a continuación los contenidos lingüísticos y culturales hallados sobre las palabras de la muestra.

\section{Análisis lingüístico y cultural de la muestra de palabras}

Baguette. Por medio del análisis de los datos, detallamos características esenciales y distintivas del concepto baguette: 
À sa naissance, elle mesurait 40 centimètres et pesait 300 grammes, contre 80 centimètres pour 250 grammes aujourd`hui (TV5 Monde, 2018, en línea).

Baguette: the standard long, crispy French loaf weighing 250g. Ficelle: a thinner, crustier $200 \mathrm{~g}$ version of the baguette [...]. Flûte: similar to, though often heavier than, a baguette (Lonely Planet, 2000, p.35).

Baguette's improved version is called the tradition, crustier, browner, and usually tastier than the classic baguette (Mas, 2014, en línea).
Croustillante, dorée et rassasiante... Aujourd'hui, la petite histoire de la baguette. Avec [...] sa croûte craquante (TV5 Monde, 2018, en línea).

Longue, fine, dorée et croustillante, la "baguette " a longtemps été considérée comme un pain de fantaisie (Chambre de métiers et de lrartisanat des Landes, 2009, p. 26).

Baguette tradition (baguette tradicional) (De Dios, 2000, p. 229).

Tabla 1

Características del concepto baguette

\begin{tabular}{cccccc}
\hline Concepto & $\begin{array}{c}\text { Características } \\
\text { esenciales }\end{array}$ & Características distintivas & \\
\hline Baguette & Pan & Alargado & $\begin{array}{c}\text { Que pesa } 250 \\
\text { gramos }\end{array}$ & $\begin{array}{c}\text { Con corteza } \\
\text { crujiente }\end{array}$ & $\begin{array}{c}\text { Con corteza } \\
\text { dorada }\end{array}$ \\
\hline
\end{tabular}

A partir de estas informaciones, una definición completa podría ser la de "pan alargado que pesa cerca de 250 gramos, con corteza dorada y crujiente". Los datos anteriores son útiles para construir árboles conceptuales en los que la palabra baguette se clasifica, interrelaciona y diferencia de otras pertenecientes al mismo campo. Basándonos en la "forma", en la figura 1 incorporamos la palabra baguette en el árbol conceptual de "pan”. 


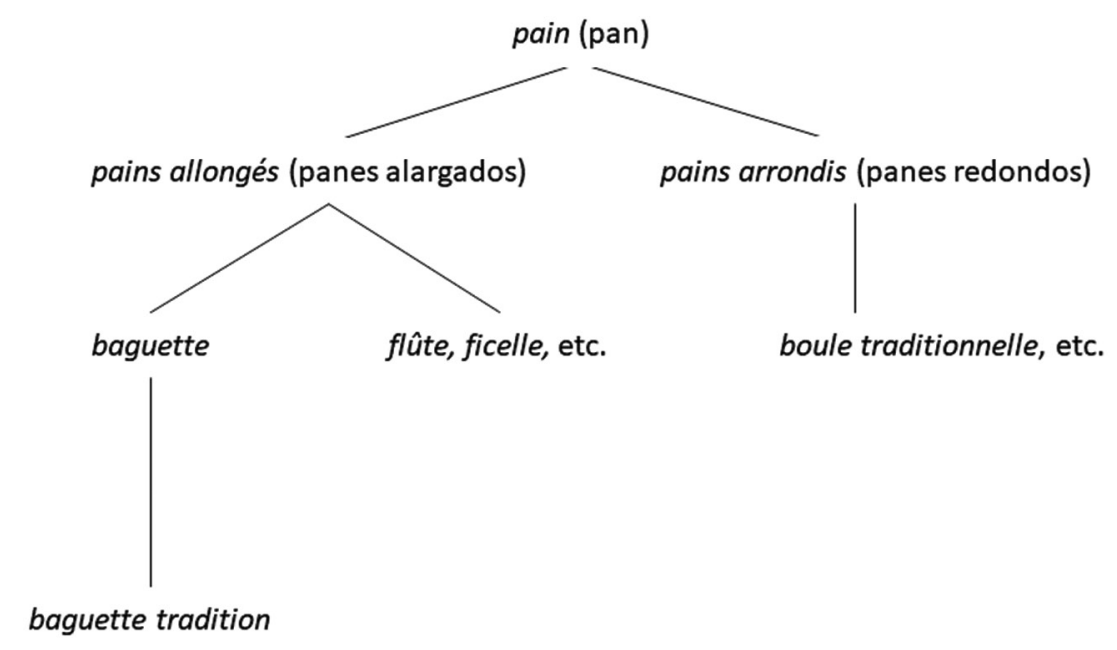

Figura 1. Árbol conceptual de la palabra "pan”.

Algunas de las fuentes también proveyeron detalles sobre otras formas de llamar a una baguette. Es el caso del sintagma baguette de pain. Se observa que la palabra baguette es una reducción de este: “S'il n'y a pas de gastronomie typiquement parisienne à part la célèbre baguette de pain" (Bourgeois y Eurin, 2001, p. 19); "Connaissent-ils le cliché du Français moyen portant un béret avec une baguette de pain à la main ?" (Augé, 2005, p. 104).

Además, hemos distinguido algunas palabras acompañadas del término baguette que son comprendidas como un elemento derivado de esta primera o como un referente diferente. La unidad lingüística demi-baguette, por ejemplo, hace referencia a la mitad de una baguette (una baguette que ha sido cortada en dos): "If you're not very hungry, ask for a demi baguette (half a loaf)" (Lonely Planet, 2000, p. 34) o a una baguette courte (que hace la mitad de la longitud de una baguette). En el segundo caso, baguette courte y demi-baguette son consideradas como unidades intercambiables: "Des baguettes courtes [...], appelées « demibaguettes "” (Paincroquant.com, 2009, en línea).

En lo que concierne a los enunciados culturales, hemos identificado varios relacionados con los modos de consumo, en qué comidas y en compañía de qué otros productos es ingerida la baguette o la demi-baguette en la actual sociedad francesa:

All boulangeries have baguettes [...] if you leave them out, they'll soon be hard - which is the way many French people like them at break-fast the next day (Lonely Planet, 2000, p. 34).

Des baguettes courtes sont parfois utilisées pour faire des sandwichs [...] On peut aussi servir une baguette courte avec du pâté ou un fromage, [...] En France, il n'est pas rare de tartiner [...] un morceau de baguette de pain avec du beurre et de la confiture, voire de 
tremper la tartine ainsi préparée dans un bol de café ou de chocolat (Paincroquant.com, 2009, en línea).

Au petit déjeuner, [...] beurre et confiture accompagnent baguette, biscottes ou croissants (Steele, 2002, p. 160).

Enfin [...] encore aujourd'hui entre tartines, sandwichs et morceaux de pain pendant les repas, on ne voit vraiment pas comment on pourrait se passer de notre petit pain quotidien (Paris ZigZag, 2016, en línea).

Aliment caméléon, il s'adapte aux nouveaux modes de vie : l'offre sandwich baguette contribue de manière croissante à la consommation de pain des Français. Les boulangers ont compris ce plébiscite aussi bien pour le traditionnel « baguette jambon beurre " que pour des compositions plus innovantes (Planétoscope, 2012a, en línea).

Se constata pues que la baguette es un alimento indispensable y central de las comidas francesas y del modelo alimentario francés.

Ocurre lo mismo con las informaciones históricas sobre las eventuales representaciones simbólicas que le han sido asignadas a esta palabra y que pueden servir para construir explicaciones o notas culturales. Así, las probables razones que permiten comprender la importancia del consumo de la baguette en Francia han sido reconocidas. Ciertas notas precisan el lugar que este producto ocupaba antes en Francia, su posible origen y el estatus que adquirió a lo largo del tiempo:
La baguette était considérée comme un pain fantaisie, privilège de l'aristocratie (TV5 Monde, 2018, en línea).

La baguette représente l'époque de la Révolution française. La Convention fait paraître un décret pour que tous les citoyens se nourrissent du même pain, tant les riches que les pauvres ; les paysans étaient réticents au changement. Plus tard, Napoléon essaie d'imposer un pain réglementaire long de $40 \mathrm{~cm}$ et pesant environ 300 g. Ce ne sera qu'après la Libération que la baguette, phénomène parisien au départ, s'imposera vraiment (Augé, 2005, p. 92).

L'origine de la baguette de pain blanc remonterait à l'époque de Napoléon. Ses boulangers auraient inventé une miche allongée pour rendre le pain plus facilement transportable par les soldats. Déjà à l'époque, le pain, c'était sacré ! (Paris ZigZag, 2016, en línea).

BAGUETTE : pain allongé [...] qui a remplacé au $\mathrm{XX}^{\mathrm{e}}$ siècle la « boule » traditionnelle (Bourgeois y Eurin, 2001, p. 132).

La baguette [...] est un pain de ville, parisien de surcroît, qui s'est popularisé depuis le début du siècle (Hubert-Baré et al., 1992, p. 206).

Las representaciones simbólicas y sociales relacionadas con la baguette han evolucionado a lo largo de la historia. Esta pasó de ser un alimento exclusivo de las clases sociales altas a uno de consumo generalizado. Además, ciertos cambios continúan presentándose. 
Así, por ejemplo, el pan negro o pan de centeno que antes era consumido por los más pobres, hoy en día, es mejor valorado que la baguette o el pan blanco, desde un punto de vista nutricional y de salud.

El valor atribuido a la baguette traspasó las fronteras y dio origen a ciertos estereotipos que los extranjeros tienen sobre los franceses:

Dans le monde, la baguette est un des symboles typiques de la France et plus particulièrement de Paris. Au même titre que le vin, le béret ou le fromage, elle est devenue un symbole alors même qu'il existe d'autres variétés de pains en France (Paincroquant.com, 2009, en línea).

La baguette sous le bras du Français moyen, coiffé de surcroît d'un béret, a été longtemps à l'étranger la caricature par excellence de l'habitant de l'Hexagone (Cuisine réponses, 2004, p. 78).

La baguette es un símbolo tan respetado como la Tour Eiffel o el Metro (De Dios, 2000, p. 128).

Estos estereotipos han sido transmitidos en gran medida por los medios de comunicación y pueden ser positivos o negativos. No hacen alusión a la raza o al sexo, sino más bien a alimentos u objetos característicos de la sociedad francesa.

Ahora bien, incluso si en Colombia ya se encuentra la baguette por un fenómeno de globalización y de préstamo alimentario, esta no posee la misma historia, connotaciones y representaciones que en Francia.
Finalmente, puesto que es evidente que la baguette no pertenece a la cultura colombiana, para traducir esta palabra se ha realizado un préstamo directo de la palabra francesa baguette. Es igualmente común escuchar la palabra "pan baguette".

Crêpe y galette. Entre las informaciones de estos conceptos, hemos identificado definiciones o datos descriptivos que especifican características para crear una más completa. Estas últimas se relacionan con la forma, los ingredientes que entran en su composición y con el modo de cocción de estos alimentos:

A large thin pancake made with white flour (Sinclair, 1998, en línea).

La crêpe est [...] une très fine couche de pâte à base de farine de blé, généralement de forme ronde, cuite sur les deux faces (Matabosch, 2009, p. 9).

la crêpe. Ce disque doré (Alimentation-France.com, 2006c, en línea).

la crêpe (ou galette) de froment ou de sarrasin (Bourgeois y Eurin, 2001, p. 97).

Les unes de blé noir, [...]. Les autres de froment (Morand, 1998, p. 225).

les crêpes, à base de farine de froment ou de sarrasin, de lait et d'œufs (Alimentation-France.com, 2006d, en línea).

crêpes ressemblent fortement aux galettes, mais ces dernières qui sont faites à partir de sarrasin (Alimentation-France.com, 2006b, en línea). 
A partir de los enunciados precedentes, podemos decir que las crêpes y las galettes se caracterizan por ser delgadas, redondas y estar hechas con harina, huevos y leche. La diferencia principal es que las primeras son preparadas con farine de froment (harina blanca) y las segundas con blé noir (harina de trigo negro). La tabla 2 resume varias características comunes y diferenciales de estos dos conceptos:

Tabla 2

Características esenciales y diferenciales de los conceptos crêpe y galette

\begin{tabular}{ccccc}
\hline \multirow{2}{*}{ Concepto } & \multicolumn{2}{c}{ Características esenciales } & \multicolumn{2}{c}{ Características diferenciales } \\
\cline { 2 - 5 } & $\begin{array}{c}\text { Delgada capa } \\
\text { de masa }\end{array}$ & $\begin{array}{c}\text { De forma } \\
\text { circular }\end{array}$ & $\begin{array}{c}\text { A base de hari- } \\
\text { na blanca }\end{array}$ & $\begin{array}{c}\text { A base de harina de } \\
\text { trigo negro }\end{array}$ \\
\hline \multirow{2}{*}{ Crêpe } & + & + & + & - \\
Galette & + & + & - & + \\
\hline
\end{tabular}

Otros de los datos obtenidos nos indican el origen geográfico de estas comidas y las posibles razones por las cuales se convirtieron en platos particulares de una región de Francia:

Une autre spécialité bretonne est la crêpe (ou galette) de froment ou de sarrasin (Bourgeois y Eurin, 2001, p. 97).

Breton Crêpes are Brittany's traditional staple, and ubiquitous throughout the region (Williams et al., 2010, p. 142).

Saviez-vous que le sarrasin qui entre dans la composition des galettes [...], fut ramené d'Asie, au XII ${ }^{\mathrm{e}}$ siècle par les croisés ? Il trouva dans les terres acides de Bretagne un milieu favorable (Alimentation-France.com, 2006b, en línea).
La harina, ingrediente primordial que entra en la composición de crêpes y galettes, así como la región de las que ellas son representativas, dan la posibilidad de crear sintagmas variables que son considerados como sinónimos. A modo de ejemplo, nombramos los siguientes: crêpe de froment, galette de sarrasin, galette bretonne $y$ galette de blé noir. También citamos otras palabras y expresiones que forman parte del campo conceptual de crêpes y galettes: crêpe Suzette, galette complète, crêpe dentelle, crêperie, crêpière, dimanche crêpier, faire sauter la crêpe, etc.:

Les adultes ont une préférence pour les « crêpes Suzette " qui sont flambées avec un alcool fort (Roesch y Rolle-Harold, 2001, p. 30).

crêpes Suzette [...], simmered in a buttery orange sauce, flamed with 
brandy and served as a dessert (Sinclair, 1998, en línea).

la fameuse "crêpe Suzette " [...] s'agissait de crêpes parfumées à l'écorce d'orange et au Grand-Marnier (Matabosch, 2009, p. 9).

les recettes avec du fromage sont des classiques. La galette complète (jambon, fromage, œuf) fait un repas complet! (Lacrampe et al., 2012, p. 71).

sauf bien sûr dans le cas des crêpes dentelles qui sont fabriquées exclusivement par des biscuiteries de Quimper (Vielfaure, 1984, p. 137).

crêpe dentelle [...] A very thin pancake from Brittany (Sinclair, 1998, en línea).

crêperie [...] A restaurant or shop usually with a connection with Brittany which specializes in filled sweet and savoury crêpes and pancakes (Sinclair, 1998, en línea).

Veillez à ce que votre crêpière ou votre poêle soit en bon état sinon vos crêpes vont attacher (Luguet-Saboulard, 2012, p. 36).

plaque de cuisson permettant d'étaler la pâte pour qu'elle soit la plus fine possible (Alimentation-France. com, 2006a, en línea).

" dimanche crêpier ", le fermier allait offrir des crêpes au propriétaire et à sa famille (Vielfaure, 1984, p. 46).

En effet, les paysans faisaient sauter la première crêpe avec la main droite tout en tenant une pièce d'or ou d'argent dans la main gauche (Matabosch, 2009, p. 17).

Otras informaciones culturales de las palabras crêpe y galette se relacionan con la forma de consumo de estos alimentos y con sus acompañamientos en la cocina francesa:

Unlike the rolled-up crêpes sold at stalls on Paris' street corners, Breton crêpes are folded envelope-style at the edges, served flat on a plate and eating using cutlery (Williams et al., 2010, p. 142).

les crêpes [...] se mangent surtout avec des accompagnements sucrés (Alimentation-France.com, 2006b, en línea).

fourrées de confiture ou de crème, flambées ou non, les crêpes (Alimentation-France.com, 2006d, en línea).

salées ou sucrées, sont célèbres et s'accompagnent de cidre, une boisson légèrement alcoolisée à base de pommes (Roesch y Rolle-Harold, 2001, p. 37).

It is famous for its cider, a sparkling alcoholic beverage made from apples and drunk with the crêpes (pancakes) from Brittany (Le Ru, 2006, p.13).

Une autre spécialité bretonne est la crêpe (ou galette) de froment ou de sarrasin, qui peut constituer un repas complet dans les nombreuses « crêperies » (Bourgeois y Eurin, 2001, p.19).

les crêpes constituent un excellent dessert; salées, enrichies de jambon, 
de champignons, de roquefort, etc., ce sont des entrées consistantes et même, souvent, un plat (Alimentation-France.com, 2006d, en línea).

Crêpes are served with variety of fillings. In Brittany, crêpes are traditionally served with cider (Eton Institute, 2012, en línea).

La crêpe autrefois, se mangeait au beurre et à la main. [...]. Le temps passant, les crêpières, pour satisfaire leur clientèle imaginèrent de les garnir avec différents ingrédients (Morand, 1998, p. 226).

Apreciamos que las crêpes son, a menudo, comidas con alimentos dulces. Las galettes, por el contrario, lo son con acompañamientos salados y las dos son frecuentemente consumidas con sidra. Finalmente, mientras que las crêpes son generalmente consideradas como postres, las galettes son, en ocasiones dadas, valoradas como plato principal.

Ahora bien, en lo que tiene que ver con la historia de estos productos, se encontraron algunos enunciados:

L'origine de la galette remonte à 7000 ans avant Jésus-Christ (Alimentation-France.com, 2006b, en línea).

Alors qu'il prépare des crêpes pour le Prince de Galles, [...], l'alcool avec lequel Charpentier les arrose s'enflamme. Comme il en a l'habitude, il continue de saupoudrer les crêpes avec du sucre qui se caramélise. Cette préparation spectaculaire et délicieuse plaît au Prince, et Charpentier se propose alors de lui dédier l'appellation. [...], le futur roi suggère plutôt qu'elles portent le prénom de sa compagne du moment "Suzette " (Neirinck y Poulain, 2000, p. 146).

Otros detalles se relacionan con las representaciones sociales y con su evolución temporal. Así, por ejemplo, la galette era vista como simple comida campesina:

la galette n'était qu'une simple bouillie étalée et desséchée (Alimentation-France.com, 2006b, en línea).

À cette époque, la galette était l'alimentation de base dans les campagnes (Alimentation-France.com, 2006b, en línea).

Plus récente que celle de sarrasin, la crêpe de froment est toutefois centenaire. L'aisance gagnant les campagnes, les paysans purent enfin utiliser la farine de froment jadis réservée aux malades et aux riches bourgeois (Morand, 1998, p. 225).

la consommation de la crêpe est relativement récente car avant le $\mathrm{XIX}^{\mathrm{e}}$ siècle, il n'est pas question, à la campagne du moins, de farine de froment. Non pas que cette denrée n'existe pas, mais la majeure partie des paysans est trop pauvre pour l'utiliser (Morand, 1998, p. 215).

Hacemos igualmente énfasis en datos sobre estas comidas conectados con mitos, creencias o supersticiones:

les crêpes seraient la survivance d'un mythe remontant aux temps les plus anciens ; elles symboliseraient la roue solaire (Villaines y Champs, 2002, p. 167). 
Autrefois, en Île-de-France et en Vendée, on conjurait la misère en déposant une crêpe au-dessus de l'armoire [...], symbole de la richesse du foyer (Lesacher, 1996, p. 18).

Souvent, pour bénéficier de cette chance toute l'année, on plaçait une crêpe sur le haut de la plus haute armoire de la maison (Vielfaure, 1984, p. 38).

Si l'on a une pièce dans la main, qu'on arrive à faire sauter la crêpe pour la retourner sans qu'elle ne reste collée au plafond, on sera riche toute l'année (Villaines y Champs, 2002, p. 167).

D'ailleurs, en faisant les crêpes, il faut respecter une autre coutume, celle de la pièce d'or. En effet, les paysans faisaient sauter la première crêpe avec la main droite tout en tenant une pièce d'or ou d'argent dans la main gauche. Ensuite, la pièce d'or ou d'argent était enroulée dans la crêpe avant d'être portée en procession par toute la famille jusque dans la chambre où on la déposait en haut de l'armoire jusqu'à la chandeleur suivante. Puis le maître de maison récupérait les débris de la crêpe de l'année précédente pour donner la pièce d'or au premier pauvre venu. Si tous ces rites étaient respectés, la famille était assurée d'avoir de l'argent toute l'année (Matabosch, 2009, p. 17).

Además, también hemos identificado que estos alimentos establecieron lazos profundos con rituales bien precisos como el Mardi gras (martes de carnaval) o La Chandeleur (fiesta de la Candelaria):
Mardi-Gras : on prépare les crêpes (Pécheur y Girardet, 2005, p. 83).

At Chandeleur (Candlemas, marking the Feast of the Purification of the Virgin Mary) on 2 February, family and friends gather together in their kitchens to make crêpes de la Chandeleur (sweet Candlemas pancakes) (Williams et al., 2010, p. 348).

Sin embargo, incluso si la asociación entre estas comidas y celebraciones continúa presentándose, su valor simbólico religioso ha desaparecido en beneficio de prácticas puramente gastronómicas y de cohesión social: "Une certitude, à l'heure actuelle la tradition des crêpes est, au cour de l'hiver, un grand moment convivial. Son sens religieux profond est complètement oublié » (Lesacher, 1996, p. 18).

Los lazos tejidos alrededor de estos alimentos son asimismo ejemplificados con la existencia de festividades particulares: "Tous les ans depuis 1993, le dernier week-end de juillet, se déroule la "Fête de la crêpe "" (Mairie de Gourin, 2018, en línea).

Si bien estas comidas no son propias de la cultura colombiana, en la actualidad, por el fenómeno de préstamo cultural alimentario, es factible encontrarlas en algunos lugares del país, en locales comerciales especializados en su venta.

Para terminar, distinguimos equivalentes de estas palabras en español. Se puede recurrir a la utilización de préstamos lingüísticos o de traducciones literales. También se pueden redactar glosas que definen o explican estos conceptos. Presentamos algunas a manera de ejemplo en la tabla 3. 
Tabla 3

Traducciones, préstamos y glosas en español de las palabras crêpe, crêpe Suzette, crêperie y galette

\begin{tabular}{ccc} 
Concepto & Traducción préstamo & Glosas \\
\hline Crêpe & Crepe & $\begin{array}{l}\text { Panqueque redondo y delgado hecho a base de ha- } \\
\text { rina, leche, huevos cocinado en una paila o crepera. }\end{array}$ \\
Crêpe Suzette & Crepe Suzette & Crepe aromatizada con jugo de naranja, azúcar y licor. \\
Galette & Crepería & Lugar en donde se preparan y se venden crepes. \\
& Galette & $\begin{array}{l}\text { Crepe de trigo negro. Crepe salada hecha con hari- } \\
\text { na de trigo negro, también llamado alforfón. }\end{array}$ \\
\hline
\end{tabular}

Soupe. Partiendo de una definición sobre este alimento: platos, más o menos caldosos, [...], con [...] elementos harinosos (pan, fideos, arroz, sémola...), hortalizas, carnes, aves, pescados o mariscos, en los que predomina el sabor del género principal (Alarcón et al., 2003, p. 204).

Distinguimos ciertos hipónimos. Los primeros se asocian al hiperónimo soupe (sopa) porque sugieren variedades en las que sobresale un ingrediente. En las dos lenguas, la estructura recurrente de las expresiones es "palabra clave, preposición " de ", sustantivo" (p. ej. soupe de légumes, sopa de verduras; soupe de poisson, sopa de pescado; soupe de vermicelles, sopa de fideos; soupe de riz, sopa de arroz). La estructura "palabra clave, artículo contracto, sustantivo" se contempla adicionalmente en francés (p. ej. soupe à la tomate, soupe à l'ail, soupe au poulet, soupe aux pois cassés).

Las sopas mencionadas poseen equivalentes en ambas lenguas y los ingredientes evocados existen en las dos zonas geográficas. No obstante, no ocurre lo mismo con el hipónimo "sopa de guineo" o "sopa de colicero" cuyo constituyente básico es un plátano verde de tamaño pequeño que se da en Colombia más no en el país europeo.

Los segundos hipónimos se vinculan al hiperónimo en cuestión al caracterizarse por su consistencia o modo de elaboración (p. ej. bouillon, caldo; crème, velouté, crema; consomé, consomé).

Algo curioso que destacamos es la expresión francesa soupe aux cailloux que implica una técnica de preparación del alimento:

La soupe aux cailloux, c'est l'une des plus fameuses recettes paysannes traditionnelles françaises. Il s'agit d'un potage de légume dans lequel les anciens rajoutaient des pierres pour qu'une fois la préparation arrivée à ébullition, elles se mettent en mouvement, broyant les légumes (France montagnes, 2016, en línea).

La palabra soupe (sopa) engloba también el léxico de las sopas de origen 
industrial. En las lenguas, señalamos las estructuras "palabra clave, preposición, sustantivo" o "palabra clave, adjetivo" (p. ej. soupe en sachet, sopa de sobre; soupe instantanée, sopa instantánea; soupe déshydratée, sopa deshidratada). Podemos contraponerlas con la expresión soupe maison (sopa casera).

Las sopas industriales fueron propuestas por el sector alimentario para satisfacer las necesidades de las comunidades cuyos estilos de vida y formas de consumir se vieron modificados al disponer de poco tiempo para cocinar. La compra de esta clase de sopas es muy frecuente en Francia:

Chaque année, un Français consomme en moyenne 12,7 litres de soupe industrielle [...] les potages déshydratés à cuire sont les plus achetés en France. [...] Les briques squattent la deuxième marche du podium [...]. Viennent ensuite les potages instantanés [...]. Et enfin, moins pratiques peut-être, les boîtes (Planétoscope, 2012b, en línea).

Otros hipónimos de la palabra soupe (sopa) aluden a platos tradicionales que representan el acervo culinario de cada país (p. ej. bouillabaisse, garbure, pot-au-feu, soupe à l'oignon, soupe aux choux, soupe au pistou, "mazamorra chiquita", "ajiaco", "sancocho"):

La garbure est une soupe typique des Pyrénées à base de choux et de pommes de terre (RegionFrance. com, 2015, en línea).

Certains potages font partie intégrante de notre patrimoine culinaire français, à l'instar de la soupe aux choux ou encore la soupe à l'oignon gratinée. [...] la soupe au pistou, à l'accent du sud (Le journal des femmes, 2018, en línea).

La mazamorra chiquita es un plato típico de la región cundiboyacense; [...] compuesta por varios productos regionales, como el maíz, las habas, cubios, papas, cilantro y ajo (DosConSentidos, s. f., en línea).

En lo tocante a palabras pertenecientes a la misma familia, citamos la unidad lingüística souper en francés. Esta es empleada con el significado de dîner (cenar) en algunas regiones del país.

Ahora bien, en compañía de la palabra clave, los verbos manger (comer) y boire (tomar) son usados para explicitar la acción que representa la ingesta del alimento. Sin embargo, notamos que en Colombia es más común escuchar la expresión "tomar sopa" y en Francia manger de la soupe. Ponemos de relieve una explicación suplementaria sobre el uso de uno u otro verbo en Francia:

Le terme le plus couramment utilisé est bien " manger " pour accompagner la soupe ! - Cela dépend de la consistance. S’il y a des morceaux ou non. Si la soupe est très liquide, nous dirons plus facilement "boire " une soupe. Si elle est épaisse, nous la mangerons - Cela dépend aussi de la vaisselle que nous utilisons pour " manger " ou " boire " notre soupe. Nous aurons tendance à boire une soupe si elle est servie dans un bol... et plutôt " manger " une soupe si elle est servie dans une assiette (Juicy, s. f., en línea). 
Señalamos también algunas informaciones etimológicas de la unidad lingüística soupe:

Originellement - et c'était toujours vrai au XIVe et XVe siècles, - il désignait une tranche de pain. [...] Puis, second stade, " soupe " a plutôt désigné l`ensemble pain-bouillon qui a longtemps constitué l’ordinaire des repas populaires, ou même - et déjà parfois à la fin du Moyen Âge - le bouillon dans lequel on faisait tremper ce pain (Flandrin y Cobbi, 1999, p. 482).

Con respecto a las informaciones culturales y tomando en consideración la frecuencia y momentos de consumo de esta comida, constatamos que en Colombia es mucho más popular y apetecida. En esta nación, se toma sopa varias veces por semana, en cualquiera de las tres comidas diarias y en sitios tanto cálidos como fríos. En Francia, se privilegia principalmente la época de invierno, aunque en ocasiones es posible consumir sopas frías en verano:

La soupe revient en force [...], elle est le plat préféré des Français chaque hiver (Agromedia, 2014, en línea).

In the Colombian diet. We have hot soups even when it's close to $90^{\circ} \mathrm{F}-$ we love soups (McCausland-Gallo, 2006, p. 33).

Consumo principal de la sopa según la comida del día. Almuerzos: 73 \%. Desayunos: 14 \%. Cena: $13 \%$ (Extra, 2018, en línea).

La changua es una sopa [...] tradicional bogotana que se acostumbra tomar al desayuno (Guía todo, 2018, en línea).

Dependiendo de los ingredientes y la cantidad, la sopa forma parte de una comida o es el plato principal. De igual modo, en Francia, es común agregarle des croûtons (pedacitos de pan tostado) o du fromage râpé (queso rallado).

En Francia, una connotación cultural de este alimento es la de considerar a las personas que lo consumen como "pasadas de moda": "Non, la soupe n'est pas ringarde. Enfin presque pas " (Madmoizelle, 2012, en línea).

La sopa también es vinculada a la pobreza: "La soupe était le repas de base chez les paysans et les gens de basses classes " (Monsieur Mada.me, 2016, en línea).

En Colombia, algunos habitantes optan por preparar sopas con ingredientes de bajo precio que posibilitan alimentar a más personas agregando más cantidad de agua. Ejemplos de estas son la "sopa de hueso" o la "sopa de menudencias" que se compone de vísceras, pescuezos o patas de gallina (Ardila et al., 2013, p.7).

En Francia, la expresión soupe populaire alude al lugar en donde se distribuye sopa u otras comidas a los más necesitados:

De 1932 à 1935, si vous étiez passés par la place Carnot, un rassemblement journalier d'hommes en casquette aurait attiré votre attention. Ils étaient là, sagement alignés, attendant devant une construction [...] d'où s'exhalait une odeur de soupe. C'est bien un bol de soupe qu'attendaient ces hommes, réunis par la misère en ce lieu qu'on appelait "La soupe populaire " (Laplace, 2015, en línea). 
Vague de froid : le triste succès de la soupe populaire, qui ne désemplit pas (Jeanjean, 2018, en línea).

Finalmente, añadimos que las sopas pueden ser el resultado de influencias o mezclas culturales: "el puchero, el cocido y el sancocho son derivaciones de la 'olla podrida', donde se mezclaron elementos indígenas y españoles" (Restrepo, 2005, p. 107).

Déjeuner. Aunque este concepto se manifiesta en los dos universos culturales estudiados (déjeuner y almuerzo), hay justamente que poner de relieve varias divergencias. Estas atañen primordialmente a los tipos de alimentos ingeridos, los acompañamientos, el número de componentes y sus modos de consumo.

Comparando el almuerzo de los sistemas culturales alimentarios colombiano y francés, observamos que en estos países la comida del mediodía está constituida de acompañamientos y bebidas específicas. En Francia, es el pan, el agua o el vino (algunas veces). En Colombia, es el arroz y el jugo de frutas:

The salade composée is the mainstay of the café and bistro lunch menu. It is a meal on a plate, and a perfect foil for a ballon de rouge, a glass of simple red wine, and a basket full of crusty bread (Herrmann, 2015, en línea).

L'eau est la première boisson inscrite au palmarès des consommations de boissons des Français. Une explication possible à la prédominance de l'eau viendrait de nos traditions culinaires héritées du XVII ${ }^{\mathrm{e}}$ siècle qui ne mettent pas en avant le mélange des saveurs sucrées et salées (Hébel, 2012, p. 2).
En France, et contrairement à d'autres pays, on ne mélange pas les saveurs sucrées et salées lors des repas principaux, tradition culinaire expliquant que l'on consomme majoritairement de l'eau à table et très faiblement des boissons rafraîchissantes au goût sucré (Hébel, 2012, p. 43).

Es típico de Colombia que el plato fuerte se acompañe de arroz y papa o yuca, o los tres juntos. [...] gracias a nuestra riqueza natural, los jugos naturales y dulces hechos con frutas son el final favorito de un almuerzo (Knorr, 2016, en línea).

Además, en este país occidental, los alimentos se consumen por separado de una forma determinada (p. ej. l'entrée, le plat principal, le dessert, etc.):

En France, le déjeuner et le dîner se composent d'une entrée, d'un plat principal, d'un fromage et d'un dessert (Lefigaro.fr, 2018, en línea).

Déjeuner : des hors-d'œuvre variés. Un plat principal (viande ou poisson avec légumes). Une salade verte. Un choix de fromages. Un dessert (Valette y Valette, 2009, p.257).

The déjeuner (lunch), [...], may still be substantial and served in three courses (entrée, plat, fromage or dessert) or may consist of a sandwich eaten on the run (Abramson, 2007, p. 103).

Lunch consists of an entrée, most often crudités (raw vegetables such as grated carrots with a vinaigrette dressing); a main dish of fish or meal with vegetables, [...] and a 
dessert (often just a piece of fruit or a tub of low fat yoghurt) (Le Ru, 2006, p. 15).

En Colombia, los productos fríos, como la ensalada, y los alimentos calientes, como el arroz o las carnes, son servidos generalmente en el mismo plato. Un orden es sin embargo establecido durante el almuerzo. En efecto, encontramos la sopa, el seco y el jugo. El seco se compone de arroz, carne (p. ej. vaca, pollo o cerdo) o pescado, ensalada y granos (p. ej. lentejas, fríjoles rojos, arvejas, garbanzos, etc.):

En todos los grupos de edad, los alimentos que más se consumen [...] En el almuerzo: arroz, sopa, jugo, carne asada, plátano frito, agua de panela, verduras y frutas (Velásquez, 2006, p. 17).

Lunch (almuerzo) is a full-on meal consisting of soup, a main course and usually a dessert (Keeling y Jacobs, 2015, en línea).

Lunch (almuerzo) is the main meal [...], it might start with a light soup, then a piece of grilled meat served with potatoes, rice (Cathey, 2011, en línea).

En relación con la hora en la que el almuerzo es consumido, se observa una semejanza entre los dos países:

Les Français déjeunent entre midi et deux heures. Beaucoup de gens déjeunent au restaurant, au café ou à la cafétéria de l'entreprise où ils travaillent. Certaines personnes rentrent chez elles et déjeunent en famille (Valette y Valette, 2009, p. 263).
Seul le déjeuner reste à un horaire fixe : $13 \mathrm{~h}$ pour la moitié des Français. À noter que $60 \%$ des cadres, des indépendants et des professions intermédiaires le sacrifient de temps en temps (Alim'agri, 2012, en línea).

Pour le déjeuner, l'Insee a calculé qu'à 13 heures, la moitié de la France est à table (Europe1, 2016, en línea).

Lunch is between noon and about 2 P.M. The break is long enough to allow for a relaxed meal and for socializing with colleagues, friends, or family (Abramson, 2007, p. 108).

Colombia [...]. Lunch is served from 12:30 or 1 P.M. To 3 P.M. (or beyond) (Foster, 2002, p. 107).

Las variaciones en los almuerzos dependen de los alimentos que se encuentran en los diferentes contextos sociogeográficos. Estos también están sujetos a la manera en la que los alimentos se emplean o manipulan. Existen patrones similares como lo es el hecho de que el almuerzo en Francia y en Colombia se inscribe dentro de un modelo de alimentación ampliamente globalizado, caracterizado por el consumo de tres comidas diarias.

\section{Conclusiones}

Los datos recolectados hicieron posible el reconocimiento de informaciones lingüísticas que figuran normalmente en los artículos de los diccionarios monolingües o bilingües. Gracias a las fuentes bibliográficas, también se 
pudieron tomar en consideración informaciones culturales para la pequeña muestra de palabras descritas. Nos pareció importante resaltarlas, porque gran parte de ellas no se explicitan en muchos de los diccionarios destinados al aprendizaje del francés como lengua extranjera. Además, teniendo en cuenta la relación estrecha que existe entre una lengua y su cultura, estos aspectos deberían incluirse en su conjunto y no de forma aislada.

Constatamos igualmente la existencia de una gran variedad de datos culturales. Estos se relacionan con comportamientos, actitudes, representaciones simbólicas, connotaciones sociales, rituales o funcionamientos que se manifiestan en las sociedades francesa y colombiana.

Por medio del paralelo establecido entre las formas de actuar y pensar en estos dos países, se profundizan las informaciones que van a figurar en las rúbricas del diccionario cultural y que podrían clasificarse en notas culturales o puntos interculturales. El análisis contrastivo de este estudio permite ahondar en el conocimiento lingüístico y sociocultural de la lengua extranjera aprendida pero también de la lengua materna. Aunque en este análisis mostramos algunos ejemplos, estos se verificarían mejor aún con la constitución de un corpus de gran volumen en el que se encuentren más ocurrencias de las palabras o fenómenos analizados.

Por otro lado, este estudio pone de relieve ciertas semejanzas entre los sistemas alimentarios francés y colombiano. Este país latinoamericano, al haber sido una colonia española, adquirió asimismo implícitamente costumbres, comportamientos o tradiciones alimentarias del continente europeo.
Estas se mezclaron con las propias generando transformaciones y aculturaciones.

En la actualidad, los sistemas alimentarios continúan interrelacionándose mediante los medios de comunicación, los fenómenos migratorios, la importación de alimentos que antes no nos eran familiares o con la comercialización de productos a escala mundial que en situaciones dadas conduce a una homogeneización alimentaria.

\section{Bibliografía}

Abramson, J. (2007). Food culture in France. Estados Unidos: Greenwood Press.

Agromedia. (2014). Les soupes : en brique ou faites maison, un marché qui bat son plein! Recuperado de http:// www.agro-media.fr/analyse/les-soupes-en-briques-maison-marche-batplein-17146.html

Alarcón, R., González, J., Ochoa, O., Rivera, J. y Roig, C. (2003). Cocineros: temario general para oposiciones. Madrid: Mad.

Alim'agri. (2012). Les habitudes alimentaires des Français évoluent. Recuperado de http://agriculture.gouv. fr/les-habitudes-alimentaires-desfrancais-evoluent

Alimentation-france.com. (2006a). Les crêpes dentelles. Recuperado de http://www.alimentation-france. com/france/specialites/les-crepesdentelles.html

Alimentation-france.com. (2006b). Les crêpes et galettes bretonnes. Recuperado de http://www.alimentation-france.com/france/specialites/les-crepeset-galettes-bretonnes.html

Alimentation-france.com. (2006c). L'histoire de la Chandeleur. Recuperado 
de http://www.alimentation-france. com/alimentation/histoire/chandeleur.html

Alimentation-france.com. (2006d). L'histoire des crêpes. Recuperado de http:// www.alimentation-france.com/alimentation/histoire/crepes.htm

Allende, I. (2014). Afrodita: cuentos, recetas y otros afrodisíacos. Madrid: Penguin Random House.

Ardila, F., Valoyes, E. y Melo, M. (2013). Documento nacional hábitos y prácticas alimentarias: hallazgos nacionales a partir del análisis departamental PAE-UNAL. Recuperado de https://www.mineducacion. gov.co/1759/articles-336866_archivo_pdf_UNAL_habitos_alimentarios.pdf

Aron, J. (1997). Cultures, nourriture. París: Maison des cultures du monde.

Augé, H. (2005). Tout va bien 1 : méthode de français, livre du professeur. París: CLÉ international.

Bourgeois, R. y Eurin, S. (2001). La France des régions. Grenoble: Presses universitaires de Grenoble.

Cathey, K. (2011). The essential guide to customs \& culture: culture smart! Colombia. Gran Bretaña: Bravo Ltd.

Chambre de métiers et de l'artisanat des Landes. (2009). Meilleure baguette des Landes. Artisanlandais, 86, pp. 1-28. Recuperado de http:// www.cma-landes.fr/file_pdf/AL/ AL\%2086\%20WEB.pdf

Cuisine réponses. (2004). Cuisine réponses : tout pour réussir l'aménagement de votre cuisine. París.

De Dios, H. (2000). Guía completa de París. Buenos Aires: Guías de Dios.

Dosconsentidos. (s.f.). Mazamorra chiquita. Recuperado de https://dosconsentidos.com/receta/mazamorra-chiquita
Eton Institute. (2012). French phrasebook: what's your next language? S.L.: Eton Institute.

Europe1. (2016). Le repas "à la française " perdure. Recuperado de http:// www.europe1.fr/france/le-repas-ala-francaise-perdure-1272213

Extra. (2018). Aunque a algunos no les gusta, aumenta el consumo de sopa en el pais. Recuperado de http://bogota.extra.com.co/noticias/ econom\%C3\%ADa/aunque-algunosno-les-gusta-aumenta-el-consumode-sopa-en-443031

Fieldhouse, P. (1995). Food and nutrition: customs and culture. Dordrecht: Springer Science + Business Media.

Fischler, C. (1990). L’homnivore. París: Odile Jacob.

Fischler, C. (1995). El (h)omnívoro: el gusto, la cocina y el cuerpo. Barcelona: Anagrama.

Flandrin, J.L. y Cobbi, J. (1999). Tables d'hier, tables d'ailleurs : histoire et ethnologie du repas. París: Odile Jacob.

Foster, D. (2002). The global etiquette guide to Mexico and Latin America: everything you need to know for business and travel success. Nueva York: John Wiley \& Sons, Inc.

Fox, R. (2003). Food and eating: an anthropological perspective. Recuperado de http://www.sirc.org/publik/foxfood.pdf

France Montagnes. (2016). 6 recettes de montagne incontournables. Recuperado de https://www.france-montagnes. com/webzine/gastronomie/6-recettesde-montagne-incontournables

Franco, A. (2000). Por nuestro ecosistema: ja comer ancas de rana! Recuperado de https://aupec.univalle.edu.co/informes/noviembre00/ranatoro.html 
Fumey, G. (2008). Penser la géographie de l'alimentation. Vox geographica, pp. 1-9. Recuperado de http://cafe-geo. net/wp-content/uploads/geographiealimentation.pdf

Google. (2018). Google. Recuperado de https://www.google.com/?gws_rd=ssl

Google Books. (2018). Google Books. Recuperado de https://books.google.com/

Goyan, P., Sucher, K. y NahikianNelms, M. (2015). Food and culture. Canadá: Cengage Learning.

Guía todo. (2018). Changua con huevo. Recuperado de http://www.guiatodo.com.co/Comida/bogota/changua_ con_huevo

Hébel, P. (2012). Comportements et consommations alimentaires en France. París: Éditions TEC \& DOC, Lavoisier.

Herrmann, S. (2015). In a French kitchen: tales and traditions of every home cooking in France. Nueva York: Penguin Random House.

Hubert-Baré, A., Comolli, M. y Carles, M. (1992). L'héritage de la cuisine française. París: Hachette.

Hymery, N., Vasseur, V., Coton, M., Mounier, J., Jany, J., Barbier, G. y Coton, E. (2014). Filamentous fungi and mycotoxins in cheese: a review. Comprehensive reviews in food science and food safety, 13, pp. 437-456. Recuperado de https://doi.org/10.1111/15414337.12069

Jeanjean, B. (2018). Vague de froid : le triste succès de la soupe populaire. Recuperado de https://www.sudradio.fr/societe/vague-de-froid-le-triste-succes-de-la-soupe-populairequi-ne-desemplit-pas

Juicy. (s.f.). Boire ou manger une soupe? Recuperado de https://magazine. permajuice.com/boire-ou-mangerune-soupe/

Keeling, S. y Jacobs, D. (2015). The rough guide to Colombia. Londres: Rough Guides Ltd.

Knorr. (2016). El corrientazo: un almuerzo con orgullo colombiano. Recuperado de http://www.knorr. com.co/articulo/detalle/559695/elcorrientazo-un-almuerzo-con-orgullo-colombiano

Lacrampe, C., Labourdette, J. y Auzias, D. (2012). La route des fromages. París: Les nouvelles éditions de l'université.

Laplace, É. (2015). Au temps de la soupe populaire. Recuperado de https://archives.saint-etienne.fr/ article.php?laref $=1006 \&$ titre $=$ autemps-de-la-soupe-populaire

Le journal des femmes. (2018). Potage, soupe et velouté. Recuperado de https://cuisine.journaldesfemmes.fr/ recette-potage-soupe

Le Ru, C. (2006). French fare. Hong Kong: Regal Printing Limited.

Lefigaro.fr. (2018). Déjeuner et dîner : que doit-on manger lors d'un repas. Recuperado de http://sante.lefigaro. fr/mieux-etre/nutrition-pratique/ dejeuner-diner/que-doit-on-mangerlors-dun-repas

Lesacher, A. (1996). Fêtes et traditions de France. Rennes: Ouest-France.

Lévi-Strauss, C. (2003). El origen de las maneras de mesa: mitológicas III. México: Siglo veintiuno editores.

Lévi-Strauss, C. (2009). Une leçon d'anthropologie : le triangle culinaire. Le nouvel observateur hors-série, pp. 14-17. Recuperado de http://docplayer.fr/7749-Une-lecon-d-anthropologie-le-triangle-culinaire.html

Lonely Planet. (2000). World food France. Sídney: Lonely Planet Publications. 
Luguet-saboulard, A. (2012). Confitures inratables : des recettes gourmandes et vraiment faciles! París: Le duc.s éditions.

Madmoizelle. (2012). Heinz et la seule pub qui donne envie de soupe. Recuperado de http://www.madmoizelle. com/publicite-soupe-heinz-132696

Mairie de Gourin. (2018). Fête de la crêpe. Recuperado de http://www.gourin.fr/ f\%C3\%AAte-de-la-cr\%C3\%AApe.html

Mas, V. (2014). Farm to table: French phrasebook. Berkeley: Ulysses Press.

Matabosch, R. (2009). La cuisine facile, tome I : les pâtes à crêpes. S.L.: TheBookEdition.

Mccausland-Gallo, P. (2006). Secrets of Colombian cooking. Nueva York: Hippocrene Books.

Monsieur mada.me. (2016). 7 plats $d u$ pauvre devenus plats du riche. Recuperado de https://monsieurmada.me/plats-du-pauvre-devenusplats-du-riche/

Morand, S. (1998). Cuisine traditionnelle de Bretagne. París: Éditions Jean-Paul Gisserot.

Neirinck, E. y Poulain, J. (2000). Histoire de la cuisine et des cuisiniers : techniques culinaires et pratiques de table en France, du Moyen-Âge à nos jours. Cachan: J. Lanore.

Paincroquant.com. (2009). Baguette. Recuperado de http://www.paincroquant.com/baguette_(pain).php

Paris zigzag. (2016). Petite histoire de la baguette : notre pain quotidien. Recuperado de http://www.pariszigzag.fr/ histoire-insolite-paris/petite-histoirede-la-baguette-notre-pain-quotidien

Pécheur, J. y Girardet, J. (2005). Campus 1: méthode de français, livre du professeur. París: CLÉ international.

Planétoscope. (2012a). Consommation de baguette de pain en France.
Recuperado de http://www.planetoscope.com/Autre/957-consommationde-baguettes-de-pain-en-france.html Planétoscope. (2012b). Consommation de soupe par les Français. Recuperado de https://www.planetoscope.com/ restauration/1443-consommationde-soupe-par-les-francais.html

Poulain, J. (2002). Manger aujourd'hui : attitudes, normes et pratiques. $\mathrm{Pa}$ rís: Éditions Privat.

Rasse, P. y Debos, F. (2006). L'alimentation, fait total de la société de communication planétaire. Communication, 25(1), pp. 179-194. Recuperado de https://communication.revues. org $/ 1413$ ?lang=en

Real Academia Española. (2016). Definición: cocina. Recuperado de http:// dle.rae.es/?id=9aoCL5w

Regionfrance.com. (2015). La garbure : la soupe paysanne des Pyrénées. Recuperado de http://regionfrance. com/garbure

Restrepo, C. (2005). Cuadernos para la historia del Colegio Mayor de Nuestra Señora del Rosario. Colombia: Centro Editorial Universidad del Rosario.

Roesch, R. y Rolle-harold, R. (2001). La France au quotidien : préparation au DELF. Grenoble: Presses universitaires de Grenoble.

Santos, S. (2013). Los sabores de la carne: influencia desde el animal vivo hasta la cocción. Recuperado de http://www.contextoganadero.com/ cronica/los-sabores-de-la-carne-influencia-desde-el-animal-vivo-hasta-la-coccion

Sinclair, C. (1998). Dictionary of food, international food and cooking: terms from $A$ to $Z$. London: A\&C Black Publishers Ltd.

Stajcic, N. (2013). Understanding culture food as a means of communication. 
Hemispheres, 28, pp. 5-14. Recuperado de http://cejsh.icm.edu.pl/cejsh/ element/bwmeta1.element.desklight3b71a1ed-2c03-499a-8884-ab5ec51e1f56

Steele, R. (2002). Civilisation progressive du français niveau intermédiaire : avec 400 activités. París: CLÉ international.

TV5 monde. (2018). L'histoire de la baguette. Recuperado de http://apprendre.tv5monde.com/fr/apprendrefrancais/lhistoire-de-la-baguette

Valette, J. y Valette, R. (2009). Contact: langue et culture françaises. Usa: Houghton Mifflin Company.

Velásquez, Gladys (2006). Fundamentos de alimentación saludable. Colombia: Universidad de Antioquia.
Verdier, Yvonne (1969). Pour une ethnologie culinaire. L'Homme, 9(1), pp. 49-57. Recuperado de http:// www.persee.fr/doc/hom_04394216_1969_num_9_1_367018

Vielfaure, N. (1984). Fêtes, coutumes et gâteaux. Le Puy: C. Bonneton.

Villaines, B. y Champs, H. (2002). Les saisons de la vie : traditions familiales et moments privilégiés du MoyenÂge à nos jours. Tournai: La Renaissance du livre.

Williams, N., Berry, O., Fallon, S., Filou, É., Le Nevez, C., Robinson, D. y Roddis, M. (2010). Discover France: experience the best of France. S.L.: Lonely Planet. 


\section{Anexo 1}

Alcaraz, M., Escoufier, D., Gomy, C., Landier, M., Quéméner, F. y Ripaud, D. (2015). Saison 1 A1+ : cahier d'activités. París: Didier.

ATILF - CNRS y Université de Lorraine (1994). Trésor de la langue française informatisé. Recuperado de http://atilf.atilf.fr/

Augé, H. (2005). Méthode de français Tout va bien 1: livre du professeur. París: CLÉ international.

Augé, H., Canada, M., Marlhens, C. y Martin, L. (2014). Nickel ! : méthode de français 1. París: CLÉ international.

Baglieto, D. y Gloanec, A. (2011). Agenda 2 : méthode de français. París: Hachette français langue étrangère.

Baglieto, D., Girardeau, B. y Mistichelli, M. (2011). Agenda 1 : méthode de français. París: Hachette français langue étrangère.

Baglieto, D., Girardeau, B., Magne, M. y Mistichelli, M. (2014). Agenda 2 : méthode de français. París: Hachette français langue étrangère.

Batra, S. y Ranjit, M. (2016). Ailes 2 : cahier d'exercices. Nueva Delhi: New Saraswati House.

Berthet, A., Daill, E., Hugot, C. y Waendendries, M. (2014). Alter ego + A1 : cahier d'activités. París: Hachette français langue étrangère.

Berthet, A., Daill, E., Hugot, C., Kizirian, V. y Waendendries, M. (2014). Alter ego + A1 : méthode de français. París: Hachette français langue étrangère.

Blanc, J., Cartier, J.-M. y Lederlin, P. (2001). Escales 1 : méthode de français, guide pédagogique. París: CLÉ international.
Bourgeois, R. y Eurin, S. (2001). La France des régions. Grenoble: Presses universitaires de Grenoble.

Bragger, J. y Rice, D. (2004). Allons-y! Le français par étapes. Canadá: Thomson Learning Inc.

Capelle, G. y Gidon, N. (1999). Reflets 1 : méthode de français, guide pédagogique. París: Hachette français langue étrangère.

Capelle, G. y Guédon, P. (2009). Le nouveau taxi 1 : méthode de français, guide pédagogique. París: Hachette français langue étrangère.

Capelle, G. y Menand, R. (2016). Le nouveau taxi 1 : méthode de français. París: Hachette français langue étrangère.

Cartier, I., Dereeper, C., Gomy, C., Ripaud, D. y Valenza, A. (2014). Saison 2 A2+ : cahier d'activités. París: Didier.

Cervoni, B., Chnane-Davin, F. y Ferreira-Pinto, M. (2006). Entrée en matière : la méthode de français pour adolescents nouvellement arrivés, guide pédagogique. París: Hachette français langue étrangère.

Chahi, F., Huor, C., Malorey, C., Marchandeau, C., Pruvost, N., Miras, G., Poisson-Quinton, S. y Delannoy, G. (2015). Entre nous 2 : méthode de français. París: Éditions maison des langues.

Claire, M. (2013). Communication progressive $d u$ français. París: CLÉ international.

Cocton, M.-N., De Oliveira, A., Dintilhac, A. y Ripaud, D. (2014). Saison $2 A+$ : méthode de français. París: Didier.

Cocton, M.-N., Heu, É., Houssa, C., Kasazian, É., Dupleix, D. y Ripaud, D. (2015). Saison 1 A1+ : méthode de français. París: Didier. 
Cocton, M.N., Cros, I., Mraz, C. y Ripaud, D. (2015). Saison 3 B1 : méthode de français. París: Didier.

Courtillon, J., De Salins, G.D. y Guyot-Clément, C. (1994). Libreéchange 3 : livre du professeur. París: Hatier Didier.

Courtillon, J., De Salins, G.D. y Guyot-Clément, C. (1998). Libreéchange 1 : livre du professeur. París: Hatier Didier.

Daill, E. y Blin, G. (2006). Alter ego 1 : méthode de français A1, guide pédagogique. París: Hachette français langue étrangère.

Dollez, C. y Pons S. (2002). Reflets 3 : méthode de français, guide pédagogique. París: Hachette.

Dubois, A.L., Lerolle, M. y Gallon, F. (2013). Scénario 1: méthode de français. Italia: Hachette français langue étrangère.

Guédon, P. (2003). Forum 3 : méthode de français, guide pédagogique. París: Hachette français langue étrangère.

Kaneman-Pougatch, M. (1997). Café crème 1 : méthode de français, guide pédagogique. París: Hachette français langue étrangère.

Kober-Kleinert, C. y Parizet, M.L. (2012). $A B C$ Delf B1. París: CLÉ international.

Larousse. (2018). Dictionnaires bilingues. Recuperado de https://www. larousse.fr/dictionnaires/bilingues

Larousse. (2018). Dictionnaire de français. Recuperado de https://www.larousse.fr/dictionnaires/francais

Magne, M., Baglieto, D., Mistichelli, M. y Giradeau, B. (2011). Agenda 2 : cahier d'activités A2. París: Hachette français langue étrangère.

Mauchamp, N. (2004). La France de toujours : civilisation. París: CLÉ international.
Menand, R., Berthet, A. y Kizirian, V. (2016). Le nouveau taxi A2 : méthode de français. París: Hachette français langue étrangère.

Mérieux, R. y Loiseau, Y. (2004). Connexions 1 : guide pédagogique. París: Didier.

Mérieux, R. y Loiseau, Y. (2004). Connexions 1 : méthode de français. París: Didier.

Murillo, J., Tost, M. y Campà, À. (2000). Forum 1 : méthode de français, guide pédagogique. París: Hachette français langue étrangère.

Murillo, J., Tost, M. y Campà, À. (2001). Forum 2 : méthode de français, guide pédagogique. París: Hachette français langue étrangère.

Parizet, M.L. (2013). ABC Delf B2. París: CLÉ international.

Pécheur, J. y Girardet, J. (2005). Campus 1 : méthode de français, livre du professeur. París: CLÉ international.

Pécheur, J. y Girardet, J. (2005). Campus 2 : méthode de français, livre du professeur. París: CLÉ international.

Poisson-Quinton, S. (2004). Expression écrite A1. París: CLÉ international.

Poisson-Quinton, S., Maheo-Le Coadic, M. y Verne-Sirieys, A. (2005). Festival 1 : méthode de français, livre du professeur. París: CLÉ international.

Poisson-Quinton, S., Maheo-Le Coadic, M. y Verne-Sirieys, A. (2006). Festival 2 : méthode de français, livre du professeur. París: CLÉ international.

Poisson-Quinton, S. y Mimran, R. (2015). Compétences A2 : expression écrite. París: CLÉ international.

Pruvost, N., Courteaud, F., Gómez-Jordana, S., Blondel, F., Chahi, F., Caballero, G., Poisson-quinton, S., Daupras, C., Delannoy, G. y Brandel, K. (2015). Entre nous 1 : méthode de français. París: Éditions maison des langues. 
Ranjit, M. (2016). Ailes 2 : méthode de français. Nueva Delhi: New Saraswati House.

Real Academia Española. (2018). Diccionario de la lengua española. Recuperado de http://dle.rae. es/?w=diccionario

Roesch, R. y Rolle-Harold, R. (2012). Civilisation la France au quotidien. Grenoble: Presses universitaires de Grenoble.
Ros Mas, R., Brockmeier, R. y Katzaros, V. (2007). Grand dictionnaire espagnol - français, français - espagnol. París: Larousse.

Steele, R. (2002). Civilisation progressive du français, niveau intermédiaire : avec 400 activités. París: CLÉ international.

Tomalin, B. (2013). Culture smart! France. Gran Bretaña: Kuperard.

\section{Anexo 2}

Addition

Apéro

Appellation d'origine

contrôlée

Artisan de bouche

Assiette

Baguette

Barbecue

Beaujolais

Bistrot

Bœuf bourguignon

Boîte de conserve

Bouillabaisse

Boulanger

Brasserie

Brioche

Bûche de Noël

Café noir

Camembert

Canard

Carafe d'eau

Carte de vins

Cassoulet

Cerise

Chablis

Charcuterie
Chausson aux pommes

Chèque restaurant

Chips

Choucroute

Cidre

Comté

Confiture

Coquilles Saint Jacques

Couscous

Couteau

Crème fraîche

Crêpe

Crêpière

Croissant

Croque-monsieur

Crudité

Cuisine française

Cuisse de grenouille

Cuisson de la viande

Déca

Déjeuner

Dessert

Diabolo

Diète méditerranéenne

Digestif

Dinde de Noël
Dîner

Dresser la table

Eau calcaire

Éclair

Entrée

Escargot

Faire la cuisine

Faire sauter la crêpe

Fait maison

Flûte

Foie gras

Formule

Fourchette

Frite

Fruit

Galette

Garçon de café

Gaspillage alimentaire

Gâteau d'anniversaire

Goûter

Grignoter

Habitude alimentaire

Heure du déjeuner

Huître

Jus de fruit

Label bio 
Légume sec

Magret de canard

Marché paysan

Menu

Mettre la table

Moule-frite

Pain d'épice

Petit beurre

Pique-niquer

Plan de table

Plat fait maison

Plat préparé

Plateau de fromages

Pot-au-feu

Poulet rôti
Pourboire

Produit Bio

Produit de terroir

Ratatouille

Régime

Repas

Repas de famille

Restauration scolaire

Resto U

Salade verte

Sandwich jambon beurre

Sauce mayonnaise

Saucisson

Serveur

Soupe
Surgelé

Table

Tartiflette

Tartine

Terrasse de café

Velouté

Vendange

Verre d'eau

Viande de bœuf

Viennoiserie

Vin de table

Vittel

Yaourt 